\title{
Desmídias filamentosas (Conjugatophyceae) de um lago de inundação de águas pretas na Amazônia Central
}

\author{
Karla Ferreira SOUZA', Sérgio MELO² \\ RESUMO \\ Com base em 109 amostras coletadas em diferentes fases do ciclo hidrológico nos anos de 2002 a 2004 no lago Cutiuaú, um \\ ambiente de águas pretas localizado no Parque Nacional do Jaú na bacia do rio Negro, foi realizado um levantamento taxonômico \\ das espécies de desmídias filamentosas. Foram identificadas 15 espécies distribuídas em sete gêneros, sendo Desmidium C. \\ Agardh ex Ralfs, com sete espécies, o gênero que apresentou a maior riqueza específica, incluindo $D$. quadratum citado pela \\ primeira vez para o estado do Amazonas. O gênero Groenbladia Teiling apresentou três espécies, enquanto os gêneros Bambusina \\ Kützing ex Kützing, Hyalotheca Ehrenberg ex Ralfs, Phymatodocis Nordstedt, Spondylosium Brébisson ex Kützing e Teilingia \\ Bourrelly, apresentaram uma única espécie. Em relação aos períodos do ciclo hidrológico, três táxons foram registrados no \\ período de águas baixas, seis no de vazante, oito no de águas altas e 15 , ou seja, todos foram observados no período de enchente.
}

PALAVRAS-CHAVE: Desmídias, águas pretas, regiāo amazônica

\section{Filamentous desmids (Conjugatophyceae) from a floodpulse black water lake in Central Amazon}

\begin{abstract}
A taxonomic survey regarding the filamentous desmids species was carried out made based on 109 samples collected in different phases of the hydrological cycle between the years of 2002 and 2004 in Cutiuaú lake, an black water system located in Jau National Park in the Negro river basin. A total of 15 filamentous desmids species distributed in seven genera were identified. Desmidium C. Agardh ex Ralfs, with seven species, was the genera with more species richness, being D. quadratum mentioned for first time in Amazonas State. Three Groenbladia Teiling species and one of Bambusina Kützing ex Kützing, Hyalotheca Ehrenberg ex Ralfs, Phymatodocis Nordstedt, Spondylosium Brébisson ex Kützing and Teilingia Bourrelly were registered. In relation to hydrological cycle, three taxa were registered in the low water period, six in the decreasing water period, eight in the high water period and 15 , all of identified taxa, in the increasing water period.
\end{abstract}

KEYWORDS: Desmids, black water, Amazon region.

1 Instituto Nacional de Pesquisas da Amazônia, INPA - E-mail: kfs19@hotmail.com

2 Instituto Nacional de Pesquisas da Amazônia, INPA - E-mail: sergio.melo@pq.cnpq.br 


\section{INTRODUÇÃO}

As desmídias constituem um dos grupos de algas com maior riqueza de espécies nos ambientes amazônicos, entre estas destacam aquelas de hábito filamentoso, que em especial para o estado do Amazonas, são listadas e/ou descritas nos trabalhos de Thomasson (1955, 1971); Förster (1974); Uherkovich e Schmidt (1974); Uherkovich e Rai (1979); Martins (1980); Bittencourt- Oliveira (1993); Sophia \& Dias (1996); Melo et al. (2004, 2005); Souza et al. (2007); Melo \& Souza (2009).

Nos lagos de inundação amazônicos, dois fatores principais, o tipo de águas e o pulso de inundação, sensu Sioli (1950) e Junk et al. (1989), respectivamente, atuam na estrutura e dinâmica da comunidade de algas. Os ambientes de águas pretas, por exemplo, apresentam características, como baixos valores de $\mathrm{pH}$ e de condutividade elétrica, o que favorece o desenvolvimento das desmídias, segundo Brook (1981) e Coesel (1996). Por outro lado o pulso de inundação apresenta forte influência na densidade populacional das desmídias e na riqueza de espécies ao longo de um ciclo sazonal (Huszar \& Reynolds, 1997, Melo et al. 2005).

Visando avançar no conhecimento sobre as desmídias dos ambientes de águas pretas do estado do Amazonas, foi realizado o presente estudo que teve por objetivo descrever e ilustrar as espécies de desmídias filamentosas de um lago de inundaçáo localizado no Parque Nacional do Jaú, na bacia hidrográfica do rio Negro, e avaliar possíveis variaçôes na composição e riqueza de espécies ao longo de três anos de estudo.

\section{MATERIAL E MÉTODOS}

O presente estudo foi baseado em amostras coletadas no lago Cutiuaú, um lago de inundação de águas pretas, localizado no Parque Nacional do Jaú (PNJ), no estado do Amazonas, na bacia do rio Negro, distante cerca de 200 quilômetros da cidade de Manaus. O lago Cutiuaú está localizado na margem direita do rio Jaú, com o qual mantem, pelo menos, um ponto de ligaçáo perene durante todo o ano. O lago apresenta profundidade da coluna d'água variando cerca de 2,0 a 12 metros, nos períodos de águas baixas e altas, respectivamente, e é caracterizado por apresentar baixos valores de $\mathrm{pH}(3,3$ a 5,2$)$ e de condutividade elétrica $(10,8$ a $\left.68,7 \mu \mathrm{S} . \mathrm{cm}^{-1}\right)$ e por elevados valores de temperatura da água $\left(27,0-31,7^{\circ} \mathrm{C}\right)$, com base nos resultados de Souza (2008).

Para o presente estudo foram realizadas 11 coletas, em dez estaçóes de amostragens (Tabela 1) situadas na área central de cada regiáo do lago. As amostras foram coletadas no período de abril de 2002 a novembro de 2004, distribuídas nos diferentes períodos do ciclo hidrológico: enchente, águas altas, vazante e águas baixas. As coletas foram realizadas com rede de plâncton com abertura de malha de $25 \mu \mathrm{m}$, através de arrasto vertical e horizontal, sendo as amostras fixadas com solução Transeau (Bicudo e Menezes, 2006).

O material coletado foi analisado em microscópio óptico Leitz Laborlux equipado com objetivas de vários aumentos $(10,25,40$ e 100x), lente micrometrada e câmara clara acopladas ao sistema óptico do microscópio. Os desenhos foram feito em câmara clara e posteriormente recobertos com tinta nanquim. $\mathrm{O}$ sistema de classificaçấo seguido foi o adotado por Mix (1972) que inclui as desmídias na classe Conjugatophyceae e ordens Zygnematales e Desmidiales. Todas as amostras analisadas foram depositadas no Herbário do Instituto Nacional de Pesquisas da Amazônia (INPA), sendo o número de registro referentes aos diferentes períodos de amostragens e diferentes estaçáo de amostragem apresentados na tabela 2 .

\section{RESULTADOS}

Chave para identificação dos gêneros registrados

1. Região apical com tubérculos. Teilingia

1. Região apical sem tubérculos

2. Células retangulares. Spondylosium

2. Células não retangulares

3. Parede celular estriada próximo aos pólos...Bambusina 3. Parede celular sem estrias polares

4. Vista apical 4-angular Phymatodocis

4. Vista apical 2-angular

5. Célula quadrangular

6. Constrição mediana notável...........Desmidium

6. Sem constrição mediana................ Hyalotheca

5. Célula panduriforme. Groenbladia

Tabela 1 - Coordenadas geográficas das estações de coletas no lago Cutiuaú (Parque Nacional do Jaú)

\begin{tabular}{lcc}
\hline \multirow{2}{*}{ Estações de coletas } & \multicolumn{2}{c}{ Coordenadas geográficas } \\
\cline { 2 - 3 } & $\mathrm{S}$ & $\mathrm{W}$ \\
\hline 1 & $01^{\circ} 50.330^{\prime}$ & $61^{\circ} 37.735^{\prime}$ \\
\hline 2 & $01^{\circ} 50.330^{\prime}$ & $61^{\circ} 37282^{\prime}$ \\
\hline 3 & $01^{\circ} 50.775^{\prime}$ & $61^{\circ} 36.880^{\prime}$ \\
\hline 4 & $01^{\circ} 51^{\prime} 189^{\prime}$ & $61^{\circ} 37.320^{\prime}$ \\
\hline 5 & $01^{\circ} 51.038^{\prime}$ & $61^{\circ} 36.889^{\prime}$ \\
6 & $01^{\circ} 50.718^{\prime}$ & $61^{\circ} 35.912^{\prime}$ \\
\hline 7 & $01^{\circ} 50.525^{\prime}$ & $61^{\circ} 35.692^{\prime}$ \\
\hline 8 & $01^{\circ} 51.645^{\prime}$ & $61^{\circ} 37.036^{\prime}$ \\
\hline 9 & $01^{\circ} 51.335^{\prime}$ & $61^{\circ} 36.233^{\prime}$ \\
\hline 10 & $01^{\circ} 51.039^{\prime}$ & $61^{\circ} 35.838^{\prime}$ \\
\hline
\end{tabular}


ACTA

AMAZONICA $\quad$ Desmídias filamentosas (Conjugatophyceae) de um lago

de inundação de águas pretas na Amazônia Central

Tabela 2 - Dados sobre as coletas no lago Cutiuaú como dia, mês, ano, estação de coleta, número da amostra analisada e o respectivo número de registro no Herbário do INPA.

\begin{tabular}{|c|c|c|c|c|c|}
\hline Dia da coleta & Mês de coleta & Ano de coleta & Estação de coleta & Número da amostra & Registro do Herbário INPA \\
\hline 11 & 4 & 2002 & 1 & 1 & INPA 222705 \\
\hline 11 & 4 & 2002 & 2 & 2 & INPA 222706 \\
\hline 11 & 4 & 2002 & 3 & 3 & INPA 222707 \\
\hline 11 & 4 & 2002 & 4 & 4 & INPA 222708 \\
\hline 11 & 4 & 2002 & 5 & 5 & INPA 222709 \\
\hline 11 & 4 & 2002 & 6 & 6 & INPA 222710 \\
\hline 11 & 4 & 2002 & 7 & 7 & INPA 222711 \\
\hline 11 & 4 & 2002 & 8 & 8 & INPA 222712 \\
\hline 11 & 4 & 2002 & 9 & 9 & INPA 222713 \\
\hline 11 & 4 & 2002 & 10 & 10 & INPA 222714 \\
\hline 27 & 6 & 2002 & 1 & 11 & INPA 222715 \\
\hline 26 & 6 & 2002 & 3 & 12 & INPA 222716 \\
\hline 27 & 6 & 2002 & 4 & 13 & INPA 222717 \\
\hline 26 & 6 & 2002 & 5 & 14 & INPA 222718 \\
\hline 26 & 6 & 2002 & 6 & 15 & INPA 222719 \\
\hline 26 & 6 & 2002 & 7 & 16 & INPA 222720 \\
\hline 27 & 6 & 2002 & 8 & 17 & INPA 222721 \\
\hline 27 & 6 & 2002 & 9 & 18 & INPA 222722 \\
\hline 27 & 6 & 2002 & 10 & 19 & INPA 222723 \\
\hline 31 & 8 & 2002 & 1 & 20 & INPA 222724 \\
\hline 31 & 8 & 2002 & 2 & 21 & INPA 222725 \\
\hline 31 & 8 & 2002 & 3 & 22 & INPA 222726 \\
\hline 31 & 8 & 2002 & 4 & 23 & INPA 222727 \\
\hline 31 & 8 & 2002 & 5 & 24 & INPA 222728 \\
\hline 31 & 8 & 2002 & 6 & 25 & INPA 222729 \\
\hline 31 & 8 & 2002 & 7 & 26 & INPA 222730 \\
\hline 31 & 8 & 2002 & 8 & 27 & INPA 222731 \\
\hline 31 & 8 & 2002 & 9 & 28 & INPA 222732 \\
\hline 31 & 8 & 2002 & 10 & 29 & INPA 222733 \\
\hline 21 & 3 & 2003 & 1 & 30 & INPA 222734 \\
\hline 21 & 3 & 2003 & 2 & 31 & INPA 222735 \\
\hline 21 & 3 & 2003 & 3 & 32 & INPA 222736 \\
\hline 21 & 3 & 2003 & 4 & 33 & INPA 222737 \\
\hline 21 & 3 & 2003 & 5 & 34 & INPA 222738 \\
\hline 21 & 3 & 2003 & 6 & 35 & INPA 222739 \\
\hline 21 & 3 & 2003 & 7 & 36 & INPA 222740 \\
\hline 22 & 3 & 2003 & 8 & 37 & INPA 222741 \\
\hline
\end{tabular}


Tabela 2 - Continuação

\begin{tabular}{|c|c|c|c|c|c|}
\hline Dia da coleta & Mês de coleta & Ano de coleta & Estação de coleta & Número da amostra & Registro do Herbário INPA \\
\hline 22 & 3 & 2003 & 9 & 38 & INPA 222742 \\
\hline 22 & 3 & 2003 & 10 & 39 & INPA 222743 \\
\hline 23 & 6 & 2003 & 1 & 40 & INPA 222744 \\
\hline 23 & 6 & 2003 & 2 & 41 & INPA 222745 \\
\hline 22 & 6 & 2003 & 3 & 42 & INPA 222746 \\
\hline 23 & 6 & 2003 & 4 & 43 & INPA 222747 \\
\hline 22 & 6 & 2003 & 5 & 44 & INPA 222748 \\
\hline 22 & 6 & 2003 & 6 & 45 & INPA 222749 \\
\hline 22 & 6 & 2003 & 7 & 46 & INPA 222750 \\
\hline 22 & 6 & 2003 & 8 & 47 & INPA 222751 \\
\hline 22 & 6 & 2003 & 9 & 48 & INPA 222752 \\
\hline 22 & 6 & 2003 & 10 & 49 & INPA 222753 \\
\hline 11 & 9 & 2003 & 1 & 50 & INPA 222754 \\
\hline 11 & 9 & 2003 & 2 & 51 & INPA 222755 \\
\hline 11 & 9 & 2003 & 3 & 52 & INPA 222756 \\
\hline 11 & 9 & 2003 & 4 & 53 & INPA 222757 \\
\hline 11 & 9 & 2003 & 5 & 54 & INPA 222758 \\
\hline 11 & 9 & 2003 & 6 & 55 & INPA 222759 \\
\hline 11 & 9 & 2003 & 7 & 56 & INPA 222760 \\
\hline 11 & 9 & 2003 & 8 & 57 & INPA 222761 \\
\hline 11 & 9 & 2003 & 9 & 58 & INPA 222762 \\
\hline 11 & 9 & 2003 & 10 & 59 & INPA 222763 \\
\hline 12 & 11 & 2003 & 1 & 60 & INPA 222764 \\
\hline 12 & 11 & 2003 & 2 & 61 & INPA 222765 \\
\hline 12 & 11 & 2003 & 3 & 62 & INPA 222766 \\
\hline 12 & 11 & 2003 & 4 & 63 & INPA 222767 \\
\hline 12 & 11 & 2003 & 5 & 64 & INPA 222768 \\
\hline 13 & 11 & 2003 & 6 & 65 & INPA 222769 \\
\hline 13 & 11 & 2003 & 7 & 66 & INPA 222770 \\
\hline 12 & 11 & 2003 & 8 & 67 & INPA 222771 \\
\hline 12 & 11 & 2003 & 9 & 68 & INPA 222772 \\
\hline 13 & 11 & 2003 & 10 & 69 & INPA 222773 \\
\hline 9 & 2 & 2004 & 1 & 70 & INPA 222774 \\
\hline 9 & 2 & 2004 & 2 & 71 & INPA 222775 \\
\hline 9 & 2 & 2004 & 3 & 72 & INPA 222776 \\
\hline 9 & 2 & 2004 & 4 & 73 & INPA 222777 \\
\hline 9 & 2 & 2004 & 5 & 74 & INPA 222778 \\
\hline 10 & 2 & 2004 & 6 & 75 & INPA 222779 \\
\hline
\end{tabular}


ACTA

AMAZONICA Desmílias filamentosas (Conjugatophyceae) de um lago

de inundação de águas pretas na Amazônia Central

\begin{tabular}{|c|c|c|c|c|c|}
\hline Dia da coleta & Mês de coleta & Ano de coleta & Estação de coleta & Número da amostra & Registro do Herbário INPA \\
\hline 10 & 2 & 2004 & 7 & 76 & INPA 222780 \\
\hline 9 & 2 & 2004 & 8 & 77 & INPA 222781 \\
\hline 9 & 2 & 2004 & 9 & 78 & INPA 222782 \\
\hline 9 & 2 & 2004 & 10 & 79 & INPA 222783 \\
\hline 4 & 4 & 2004 & 1 & 80 & INPA 222784 \\
\hline 4 & 4 & 2004 & 2 & 81 & INPA 222785 \\
\hline 4 & 4 & 2004 & 3 & 82 & INPA 222786 \\
\hline 4 & 4 & 2004 & 4 & 83 & INPA 222787 \\
\hline 4 & 4 & 2004 & 5 & 84 & INPA 222788 \\
\hline 4 & 4 & 2004 & 6 & 85 & INPA 222789 \\
\hline 4 & 4 & 2004 & 7 & 86 & INPA 222790 \\
\hline 4 & 4 & 2004 & 8 & 87 & INPA 222791 \\
\hline 4 & 4 & 2004 & 9 & 88 & INPA 222792 \\
\hline 4 & 4 & 2004 & 10 & 89 & INPA 222793 \\
\hline 26 & 6 & 2004 & 1 & 90 & INPA 222794 \\
\hline 26 & 6 & 2004 & 2 & 91 & INPA 222795 \\
\hline 26 & 6 & 2004 & 3 & 92 & INPA 222796 \\
\hline 26 & 6 & 2004 & 4 & 93 & INPA 222797 \\
\hline 26 & 6 & 2004 & 5 & 94 & INPA 222798 \\
\hline 26 & 6 & 2004 & 6 & 95 & INPA 222799 \\
\hline 26 & 6 & 2004 & 7 & 96 & INPA 222800 \\
\hline 25 & 6 & 2004 & 8 & 97 & INPA 222801 \\
\hline 25 & 6 & 2004 & 9 & 98 & INPA 222802 \\
\hline 26 & 6 & 2004 & 10 & 99 & INPA 222803 \\
\hline 21 & 10 & 2004 & 1 & 100 & INPA 222804 \\
\hline 21 & 10 & 2004 & 2 & 101 & INPA 222805 \\
\hline 22 & 10 & 2004 & 3 & 102 & INPA 222806 \\
\hline 21 & 10 & 2004 & 4 & 103 & INPA 222807 \\
\hline 22 & 10 & 2004 & 5 & 104 & INPA 222808 \\
\hline 21 & 10 & 2004 & 6 & 105 & INPA 222809 \\
\hline 22 & 10 & 2004 & 7 & 106 & INPA 222810 \\
\hline 22 & 10 & 2004 & 8 & 107 & INPA 222811 \\
\hline 22 & 10 & 2004 & 9 & 108 & INPA 222812 \\
\hline 21 & 10 & 2004 & 10 & 109 & INPA 222813 \\
\hline
\end{tabular}




\section{DESCRIÇÕES DOS GÊNEROS E ESPÉCIES REGISTRADOS}

\section{Bambusina Kützing ex Kützing}

Células aproximadamente cilíndricas com forma de barrilete, unidas ápice a ápice constituindo filamentos levemente torcidos. Apresentam constrição mediana leve, ápice truncado com estrias longitudinais.

\section{Bambusina brebissonii Kützing ex Kützing}

$$
\text { Spec. Algar., p. } 188.1849
$$

\section{Figura 1.}

Células $20-29 \mu \mathrm{m}$ X 11-22 $\mu \mathrm{m} ; 1,2-2$ vezes mais longas que largas. Semicélula piramidal-truncada; parede celular lisa; células unidas aos ápices com pequena protuberância nos ângulos da regiáo apical; regiáo do istmo com pequena

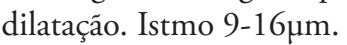

Ocorrência: amostras 1,2 , 3, 4, 5, 6, 7, 8, 9, 10, 16, 20, $21,22,24,30,31,32,33,34,35,36,37,38,39,40,44$, $54,59,67,80,81,83,84,85,86,87,88,89,92,93,95$, 96, 99, 106.

Dados abióticos: profundidade de 7,2 metros; temperatura da água $28,2^{\circ} \mathrm{C}$; concentraçáo de oxigênio de $16,3 \%$; $\mathrm{pH} 4,3$; condutividade elétrica $16,3 \mu \mathrm{S} . \mathrm{cm}^{-1}$; transparência da água 1,2 metros.

Distribuição geográfica no Amazonas: rio Negro (Thomasson, 1955); Lago Rio Preto da Eva (Thomasson, 1971); Parque Nacional do Jaú (Melo et al., 2004); lago Tupé (Melo et al., 2005); Aprile e Mera (2007); lago Cutiuaú (Melo e Souza, 2009).

\section{Desmidium C. Agardh ex Ralfs}

Células unidas aos ápices, formando filamentos torcidos. Células usualmente mais largas que longas e constrição mediana rasa; semicélulas variáveis no formato que podem ser oblongo, piramidal truncado; geralmente ápices com ligeira projeção nos ângulos.

1. Células unidas no ápice por processos curtos em cada ângulo

2. Célula quadrangular D. baileyi

2. Célula retangular. D. aptogonium var. acutius

1. Células unidas em toda região apical

3. Semicélula retangular

4. Largura do istmo $14-17 \mu \mathrm{m}$ D. quadratum

4. Largura do istmo $21-31 \mu \mathrm{m}$ ..D. coarctatum var. cambricum

3. Semicélula piramidal- truncada

5. Margem lateral reta D. elegans

5. Margem lateral arredondada 6. Largura da célula 36-45 $\mu \mathrm{m}$. D. grevillii
6. Largura da célula 22-28 $\mu \mathrm{m}$ D. cylindricum

Desmidium aptogonum Brébisson ex Kützing var. acutius Nordstedt

Minnesskr. Utg. Kongl. Fysiogr. sallsk. Lund, méd anledn. Af. Dess hundraarsfest. Pl.1, Figs. 21-22. 1878.

Figura 2.

Células $15-17 \mu \mathrm{m}$ X 26-35 $\mu \mathrm{m}$; 0,5-0,6 vez mais longas que largas. Células retangulares; seno mediano ligeiramente aberto; processos nos ângulos apicais que fazem a uniáo entre as células, resultando em uma cavidade entre as células. Istmo 10-22 $\mu \mathrm{m}$.

Ocorrência: amostras 31, 36, 39, 101.

Dados abióticos: profundidade de 5,1 metros; temperatura da água $28,4^{\circ} \mathrm{C}$; concentraçáo de oxigênio de $42,3 \%$; $\mathrm{pH} 3,9$; condutividade elétrica $18,3 \mu \mathrm{S} . \mathrm{cm}^{-1}$; transparência da água 1,2 metros.

Distribuiçáo geográfica no Amazonas: rio Jauaperi, rio Jaú (Uherkovich \& Rai,1979); lago Macurani (Sophia e Dias, 1996); lago Cutiuaú (Melo \& Souza, 2009).

Desmidium baileyi (Ralfs) Nordstedt

Acta Univ. Lund. 16: 4. 1880.

Figura 3.

Células $17-20 \mu \mathrm{m}$ X $25 \mu \mathrm{m} ; 0,8-0,9$ vez mais longas que largas. Célula quadrangular, constrição mediana ausente, margem apical com ligeira depressão; margem lateral paralela às vezes ondulada próxima ao istmo; semicélulas unidas pelos processos cilíndricos.

Ocorrência: amostras 7, 31, 32, 33, 36, 37, 38, 39, 89.

Dados abióticos: profundidade de 5,5 metros; temperatura da água $28,4^{\circ} \mathrm{C}$; concentração de oxigênio de $8,9 \%$; $\mathrm{pH} 4,1$; condutividade elétrica $22,9 \mu \mathrm{S} . \mathrm{cm}^{-1}$; transparência da água 1,2 metros.

Distribuição geográfica no Amazonas: rio Negro, Manaus (Thomasson, 1955); igarapé Laguinho, rio Maués- Assú, rio Canumẫ (Förster, 1974); rio Unini, rio Jaú (Uherkovich \& Rai, 1979); Reservatório de Balbina (Bittencourt- Oliveira, 1993); lago Macurani, rio Mamuru (Sophia \& Dias, 1996); lago Tupé (Melo et al., 2005); lago Cutiuaú (Melo \& Souza, 2009).

Desmidium coarctatum Nordstedt var. cambricum West

Jour. Roy. Microsc. Soc. 6:283. Pl.5, Fig 2. 1890.

Figura 11

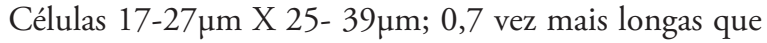
largas. Células retangular; ângulos basais arredondados; ápice plano e largo. Istmo $21-31 \mu \mathrm{m}$.

Ocorrência: amostras 5, 10, 84 
Dados abióticos: profundidade de 7,5 metros; temperatura da água $27,3^{\circ} \mathrm{C}$; concentração de oxigênio de $23,4 \%$; $\mathrm{pH} 4,3$; condutividade elétrica $17,6 \mu \mathrm{S} . \mathrm{cm}^{-1}$; transparência da água 1,2 metros.

Distribuição geográfica no Amazonas: Parque Nacional do Jaú (Melo et al., 2004); lago Tupé (Melo et al., 2005); lago Cutiuaú (Melo \& Souza, 2009).

Desmidium cylindricum Greville ex Nordstedt

The Ray Soc. London, p.249, t. 16.4:7-8.1923.

Figura 6.

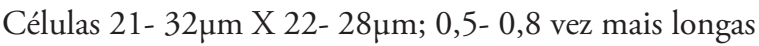
que largas. Célula quadrangular unidas em toda regiâo apical; ápice plano, istmo largo; seno mediano ligeiramente aberto. Istmo 13-38 $\mu \mathrm{m}$.

Ocorrência: amostras 32, 35, 36, 37.

Dados abióticos: profundidade de 4,6 metros; temperatura da água $28,9^{\circ} \mathrm{C}$; concentraçáo de oxigênio de $13,5 \%$; $\mathrm{pH} 5,3$; condutividade elétrica $25,2 \mu \mathrm{S} . \mathrm{cm}^{-1}$; transparência da água 1,3 metros.

Distribuição geográfica no Amazonas: Thomasson (1955); rio Cuieiras, rio Branquinho, rio Unini, rio Jaú, rio Carabinani (Uherkovich \& Rai, 1979); lago Cristalino (Martins, 1980); lago Cutiuaú (Melo \& Souza, 2009).

\section{Desmidium elegans (Raciborski) Grönblad}

Acta Soc. Sci. Fenn, II, B, 2(6):34. Pl.15, Figs. 331-333. 1945.

Figura 7.

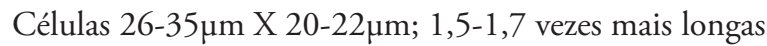
que largas. Células unidas em toda região apical. Semicélula piramidal-truncada; constriçấo mediana pouco marcada; semicélula unidas pelos ápices. Istmo 15-20 $\mu$ m.

Ocorrência: amostras 1, 2, 3, 4, 5, 6, 7, 8, 9, 10, 15, 32, 34, 60, 86.

Dados abióticos: profundidade de 6,9 metros; temperatura da água $27,8^{\circ} \mathrm{C}$; concentraçáo de oxigênio de $15,1 \%$; $\mathrm{pH} 4,5$; condutividade elétrica $13,6 \mu \mathrm{S} . \mathrm{cm}^{-1}$; transparência da água 1,1 metros.

Distribuição geográfica no Amazonas: Thomasson (1955); rio Paraconi (Förster, 1974); rio Unini, rio Carabinani (Uherkovich \& Rai, 1979); Parque Nacional do Jaú (Melo et al., 2004); lago Tupé (Melo et al., 2005); lago Cutiuaú (Melo $\&$ Souza, 2009).

\section{Desmidium grevillii (Kützingii) De Bary}

Untersuch. Fam. Conjugat. P.76. Pl. 4, Figs. 30,32. 1858.

Figuras 9a- b.

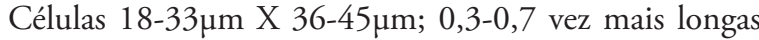
que largas. Célula retangular; ápice reto; margem celular lisa; istmo largo; vista apical elíptica. Istmo $18-38 \mu \mathrm{m}$.

Ocorrência: amostras 2, 3, 7, 30, 31, 32, 46, 81, 84, 85, 86, 87, 89.

Dados abióticos: profundidade de 7,0 metros; temperatura da água $27,9^{\circ} \mathrm{C}$; concentração de oxigênio de $25,2 \%$; $\mathrm{pH} 4,1$; condutividade elétrica $19,6 \mu \mathrm{S} . \mathrm{cm}^{-1}$; transparência da água 1,3 metros.

Distribuição geográfica no Amazonas: rio Mamuru (Sophia \& Dias, 1996); lago Tupé (Melo et al. 2005); lago Cutiuaú (Melo \& Souza, 2009).

\section{Desmidium quadratum Nordstedt}

Acta Univ. Lund, 9:49. Pl. 1, fig.24.1873.

Figura 8.

Células $15-20 \mu \mathrm{m}$ X 17-20 $\mu \mathrm{m}$; 0,7-1 vez mais longas que largas. Semicélula retangular; ápice reto; istmo largo; seno mediano levemente aberto. Istmo $14-17 \mu \mathrm{m}$.

Ocorrência: amostras 20, 32, 35, 36, 39, 83, 86, 87

Dados abióticos: profundidade de 5,8 metros; temperatura da água $28,5^{\circ} \mathrm{C}$; concentração de oxigênio de $9,4 \%$; $\mathrm{pH} 4,5$; condutividade elétrica $24,2 \mu \mathrm{S} . \mathrm{cm}^{-1}$; transparência da água 1,3 metros.

Distribuição geográfica no Amazonas: lago Cutiuaú (Melo \& Souza, 2009)

\section{Groenbladia Teiling}

Células cilíndricas, mais ou menos intumescidas na base, margems laterais usualmente paralelas entre si, ápice truncado e reto. Apresentam constrição mediana rasa e ampla.

1. Células panduriformes. G. undulata

1. Células cilíndricas

2. Células três vezes mais longas que largas G. neglecta var. neglecta

2. Células quatro vezes mais longas que largas........................ neglecta var. elongata

Groenbladia neglecta (Raciborski) Teiling var. neglecta

Bot. Notiser. p.276.1952.

Figura 12.

Células $27-38 \mu \mathrm{m} X 12 \mu \mathrm{m} ; 2,2-3$ vezes mais longas que largas. Células cilíndricas com regiáo mediana definida; ápice largo e truncado; ligeiramente constrita; células unidas nos ápices.

Ocorrência: amostras 1, 2, 7, 8, 9, 10, 13, 17, 80, 81

Dados abióticos: profundidade de 8,3 metros; temperatura da água $27,3^{\circ} \mathrm{C}$; concentração de oxigênio de $10,2 \% ; \mathrm{pH} 4,3$; 
condutividade elétrica $12,9 \mu \mathrm{S} . \mathrm{cm}^{-1}$; transparência da água 1,1 metros.

Distribuição geográfica no Amazonas: rio Negro (Uherkovich \& Rai, 1979); lago Cutiuaú (Melo e Souza, 2009).

\section{Groenbladia neglecta (Raciborski) Teiling var. elongata} Scott e Croasdale

Acta Soc. Sc. Fenn. p.48, t.35:17-24.1957.

Figura 13.

Células $42-58 \mu \mathrm{m}$ X $10-12 \mu \mathrm{m}$; 4,2-5 vezes mais longas que largas. Células cilíndricas e alongadas em relação a forma típica; ápice largo e truncado; células unidas nos ápices.

Ocorrência: amostras 1, 2, 3, 4, 5, 6, 12

Dados abióticos: profundidade de 8,3 metros; temperatura da água $27,1^{\circ} \mathrm{C}$; concentração de oxigênio de $10,9 \%$; pH 4,5; condutividade elétrica $10,6 \mu \mathrm{S} . \mathrm{cm}^{-1}$; transparência da água 1,1 metros.

Distribuição geográfica no Amazonas: rio Cuieiras (Uherkovich \& Rai 1979); Parque Nacional do Jaú (Melo et al., 2004); lago Cutiuaú (Melo \& Souza, 2009).

\section{Groenbladia undulata (Nordstedt) Förster}

Nova Hedwigia, 23(2/3):578.Pl.26, Fig.10. 1972.

Figura 10.


longas que largas. Células panduriformes com constrição mediana acentuada; ápice truncado; células unidas nos ápices para formar filamentos não torcidos. Istmo $4-7 \mu \mathrm{m}$.

Ocorrência: amostras 4, 5, 6, 11, 30, 31, 32, 33, 34,35, $36,37,38,39,41,42,43,74,76,77,78,79,82,89,101$, 104, 106, 109

Dados abióticos: profundidade de 6,5 metros; temperatura da água $29^{\circ} \mathrm{C}$; concentração de oxigênio de $21 \%$; $\mathrm{pH} 4,6$; condutividade elétrica $19,4 \mu \mathrm{S} . \mathrm{cm}^{-1}$; transparência da água 1,1 metros.

Distribuição geográfica no Amazonas: rio Maués- Assú, lago do Elias (Förster, 1974); lago Cutiuaú (Melo \& Souza, 2009).

\section{Hyalotheca Ehrenberg ex Ralfs}

Células subcilíndricas; seno mediano com leve depressão; semicélulas com margens laterais retas ou suavemente convexas. Filamentos longos com margens superiores truncadas e retas.

\section{Hyalotheca dissiliens (Smith) Brébisson ex Ralfs \\ British Desmidiaceae, p.51, t.1:1. 1848.}

Figura 14.

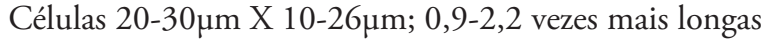
que largas. Semicélula cilíndrica, margem lateral e apical reta.

Ocorrência: amostras 34, 47

Dados abióticos: profundidade de 8,3 metros; temperatura da água $28,8^{\circ} \mathrm{C}$; concentraçáo de oxigênio de $14,9 \%$; $\mathrm{pH} 4,6$; condutividade elétrica $18,9 \mu \mathrm{S} . \mathrm{cm}^{-1}$; transparência da água 1,2 metros.

Distribuição geográfica no Amazonas: Thomasson (1955); lago do Castanho (Uherkovich e Schmidt, 1974); rio Negro, rio Jaú (Uherkovich \& Rai 1979); lago Macurani (Sophia \& Dias, 1996); rio Jaú (Díaz- Castro, 1999); lago Cutiuaú (Melo $\&$ Souza, 2009).

\section{Phymatodocis Nordstedt}

Células de contorno quadrangular em vista frontal, variando de moderada até profundamente constritas na regiáo mediana, seno mediano pode ser linear e estreito ou aberto.

Phymatodocis nordstedtiana Wolle

Bull. Torrey Bot. Club 11 (2): 15. 1884.

Figuras $15 \mathrm{a}-\mathrm{b}$.


largas. Semicélula retangular; seno mediano fechado; istmo arredondado; protuberância na região mediana; vista apical 4-angular. Istmo $10-17 \mu \mathrm{m}$.

Ocorrência: amostras 20, 32, 35, 36

Dados abióticos: profundidade de 5,9 metros; temperatura da água $29,1^{\circ} \mathrm{C}$; concentração de oxigênio de 8,9\%; $\mathrm{pH} 5,1$; condutividade elétrica $26,3 \mu \mathrm{S} . \mathrm{cm}^{-1}$; transparência da água 1,3 metros.

Distribuição geográfica no Amazonas: Thomasson (1955); lago Cutiuaú (Melo \& Souza, 2009).

\section{Spondylosium Brébisson ex Kützing}

Células de formato variável desde elíptica, oblonga a piramidal-truncada, profundamente constritas na regiáo mediana, istmo estreito e aberto.

\section{Spondylosium desmidiiforme (Borge) West e West}

J. Bot., Lond. 42: 286, PL. 464, fig. 11-12. 1904.

Figura 5.

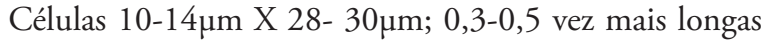
que largas. Semicélula retangular; istmo largo; margem da semicélula côncava; filamentos formados pela uniáo das células aos ápices. Istmo 24- 25 $\mu \mathrm{m}$.

Ocorrência: amostras 8, 32, 35, 36, 41, 49

Dados abióticos: profundidade de 7,5 metros; temperatura da água $28,7^{\circ} \mathrm{C}$; concentração de oxigênio de $15,6 \%$; $\mathrm{pH} 4,8$; condutividade elétrica $19,3 \mu \mathrm{S} . \mathrm{cm}^{-1}$; transparência da água 1,1 metros. 

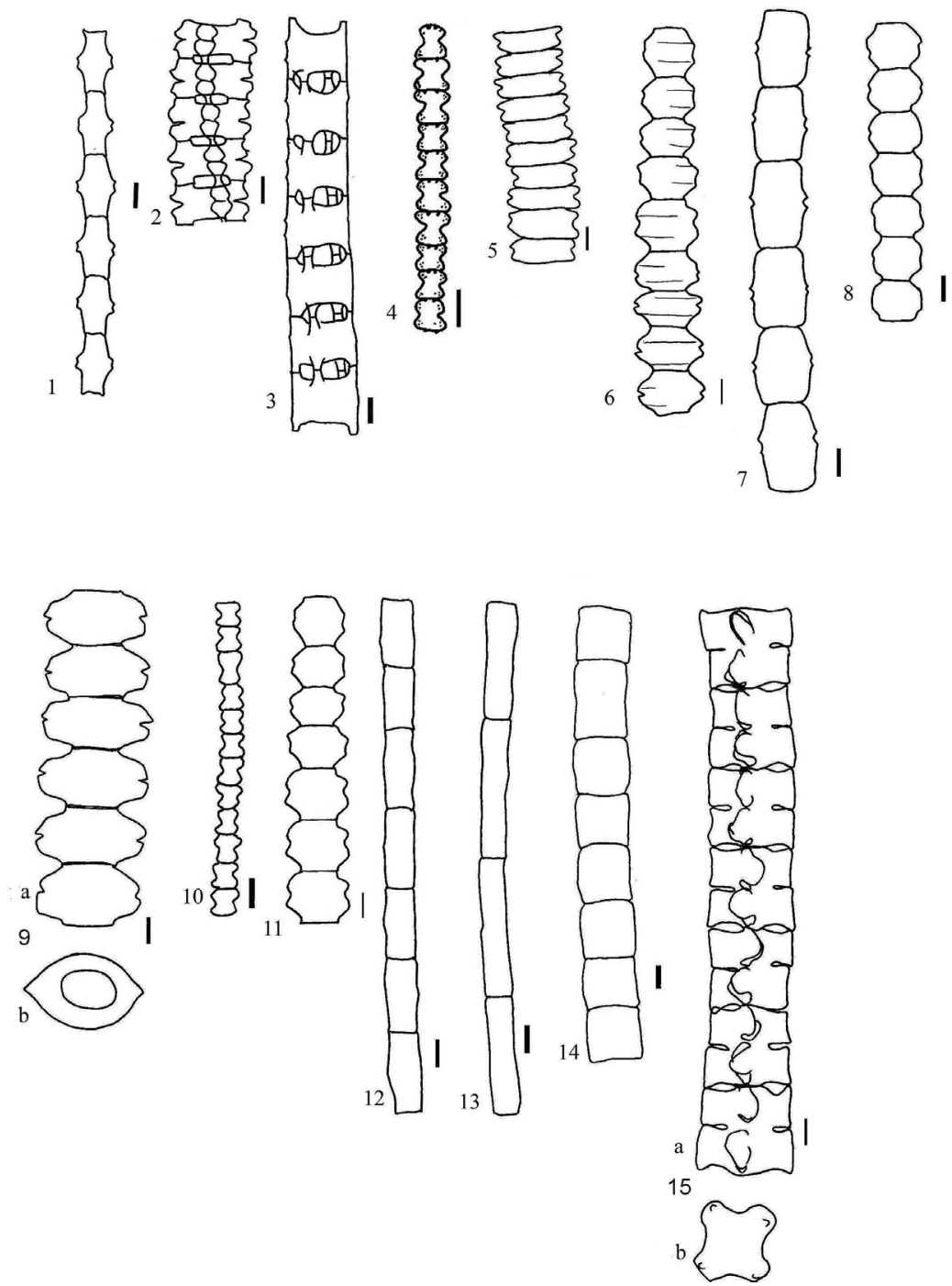

Figura 1-15 - 1- Bambusina brebissonii Kützing ex Kützing; 2- Desmidium aptogonium Brébisson var. acutius Nordstedt; 3- Desmidium baylei (Ralfs) Nordstedt; 4- Teilingia granulata (Roy \& Bisset) Bourrelly; 5- Spondylosium desmidiforme (Borge) West; 6- Desmidium cylindricum Greville ex Nordstedt; 7- Desmidium elegans (Raciborski) Grönblad; 8-Desmidium quadratum Nordstedt; 9a- b- Desmidium grevilii (Kützingii) De Bary; 10-Groenbladia undulata (Nordstedt) Förster; 11-Desmidium coarctatum Nordstedt var. cambricum West; 12-Groenbladia neglecta (Raciborski) Teiling var. neglecta; 13- Groenbladia neglecta (Raciborski) Teiling var. elongata Scott e Croasdale; 14- Hyalotheca dissiliens (Smith) Brébisson ex Ralfs; 15a- b- Phymatodocis nordstedtiana Wolle

Distribuição geográfica no Amazonas: lago Rio Preto da Eva (Thomasson, 1971); rio Maués Mirim (Förster 1974); rio Branquinho (Uherkovich \& Rai, 1979); lago Tupé (Melo et al., 2005); rio Urubuí (Aprile \& Mera, 2007); lago Cutiuaú (Melo \& Souza, 2009).

\section{Teilingia Bourrelly}

Semicélulas podem desde elípticas a quadrangulares, com constrição mediana moderada, seno mediana aberto e amplo. Presença de grânulos na margem superior.

\section{Teilingia granulata (Roy e Bisset) Bourrelly}

Revue algol., New Ser. 7 (2): 190. 1964.

Figura 4 .

Células 6-8 $\mu \mathrm{m}$ X 7-8 $\mu \mathrm{m} ; 0,8-1$ vez mais longas que largas. Célula com formato panduriforme; seno mediano aberto; base da semicélula côncava; grânulos distribuídos na margem da parede celular. Istmo $3-5 \mu \mathrm{m}$.

Ocorrência: amostras 60, 61, 62, 63, 64, 65, 67, 69, 73, 100,101 
Dados abióticos: profundidade de 2,4 metros; temperatura da água $31,5^{\circ} \mathrm{C}$; concentraçáo de oxigênio de $61,3 \%$; pH 4,6; condutividade elétrica $16,1 \mu \mathrm{S} . \mathrm{cm}^{-1}$; transparência da água 0,6 metros.

Distribuição geográfica no Amazonas: rio Maués- assú, rio Maués Mirim (Förster, 1974); lago Cutiuaú (Melo \& Souza, 2009).

\section{DISCUSSÃO}

Foram identificados 15 espécies de desmídias filamentosas, distribuídas em sete gêneros, sendo o gênero Desmidium Agardh o que apresentou a maior riqueza com sete espécies, enquanto Groenbladia Teiling apresentou três espécies, os demais gêneros registrados, Bambusina Kützing, Spondylosium Brébisson, Phymatodocis Nordestedt, Sphaerozosma Corda e Hyalotheca Ehrenberg, apresentaram apenas uma espécie. Bambusina brebissonii Kützing, Groenbladia undulata (Nordstedt) Förster e Desmidium grevilii (Kützingii) De Bary foram as espécies mais frequente, tendo sido registradas em 41,25 e $12 \%$ das amostras analisadas, respectivamente.

Em relação à variação temporal, o período de enchente foi destacado por apresentar todas as espécies registradas, sendo a enchente de 2003 a que apresentou maior número de espécies, tendo sido registradas 11 . Por outro lado, os períodos de vazante de 2003 e águas altas de 2004 com o registro de apenas um táxon, foram os períodos com menor número de espécies de desmídias filamentosas. Como mencionado por Melo et al. (2004) a maior riqueza de espécies no período de enchente parece estar relacionado a maior disponibilidade de habitat, em especial aqueles relacionados à presença de macrofítas aquáticas, que naquele período colonizam uma maior área do lago e podem servir como substrato para as desmídias, segundo Brook (1981).

Em relação à distribuição das espécies na região amazônica brasileira (Tabela 3) foi constatado que todas ocorreram no estado do Pará, quatro nos estado de Rondônia e do Mato Grosso e uma no estado do Tocantins, refletindo, provavelmente, o maior número de estudos no primeiro estado. Entre as espécies, Bambusina brebissonii foi a mais comum nos estudos, tendo ocorrido em 54,5\% deles, enquanto Desmidium coarctatum var. cambricum, Groenbladia neglecta e G. undulata, foram as menos freqüentes, ocorrendo em $9 \%$ dos trabalhos mencionados.

\section{AGRADECIMENTOS}

Os autores agradecem a Fundação Vitória Amazônica/ WWF pelo apoio logístico, ao CNPq (Proc.: 473699/20044) pelo apoio financeiro, a FAPEAM pela bolsa de mestrado concedida à primeira autora, e ao Programa de Pós-Graduaçáo em Botânica do INPA.

Tabela 3 - Distribuição geográfica dos táxons identificados em estados da região amazônica, exceto o Amazonas. Os oito primeiros referem-se ao Pará, o nono a Rondônia, 0 décimo ao Tocantins e 0 décimo primeiro ao Mato Grosso. Os números em arábico referem-se as publicações mencionadas abaixo da tabela.

\begin{tabular}{|c|c|c|c|c|c|c|c|c|c|c|c|}
\hline Espécies/Trabalhos & 1 & 2 & 3 & 4 & 5 & 6 & 7 & 8 & 9 & 10 & 11 \\
\hline Bambusina brebissonii & $\mathrm{X}$ & & $\mathrm{X}$ & $x$ & $\mathrm{X}$ & & & & $\mathrm{X}$ & & $\mathrm{X}$ \\
\hline Desmidium aptogonium var. acutius & & & $x$ & $x$ & & & & & & & \\
\hline Desmidium baylei & & & $x$ & & & & & & & & $x$ \\
\hline Desmidium coarctatum var. cambricum & & & $x$ & & & & & & & & \\
\hline Desmidium cylindricum & & & $x$ & $\mathrm{x}$ & & $x$ & $x$ & & & & \\
\hline Desmidium elegans & & & $x$ & $x$ & & & & & & & \\
\hline Desmidium grevilii & & & $x$ & $x$ & & & & & $x$ & & \\
\hline Desmidium quadratum & & & $x$ & $\mathrm{x}$ & & & & & & & $\mathrm{X}$ \\
\hline Desmidium grevilii & & & $x$ & $x$ & & & & & $x$ & & \\
\hline Groenbladia neglecta var. neglecta & & & $x$ & & & & & & & & \\
\hline Groenbladia neglecta var. elongata & & & $x$ & $x$ & & & & & & & \\
\hline Groenbladia undulata & & & & & $x$ & & & & & & \\
\hline Hyalotheca dissiliens & & & $x$ & $x$ & & & & & & $x$ & \\
\hline Phymatodocis nordstedtiana & & & & $x$ & & & & & & & \\
\hline Spondylosium desmidiiforme & & $x$ & $x$ & & & & & & $x$ & & \\
\hline Teilingia granulata & & & & & & & & $x$ & & & $x$ \\
\hline
\end{tabular}




\section{BIBLIOGRAFIA CITADA}

Aprile, F.M.; Mera, P.A.S.2007. Phytoplankton and phytoperiphyton of a black-waters river from North Peripheral Amazon. Braz. J. Aquat. Sci. Technol., 2007, 11(2):1-14 (in Portuguese, with abstract in English).

Bicudo, C.E.M.; Menezes, M. 2006. Genera of continental algae from Brazil: Identification key and descriptions. Rima: São Carlos, São Paulo. 508pp (in Portuguese).

Bittencourt-Oliveira, M.C. 1993. Algal flora reservoir, State of Amazonas, III: Cass Zygnemaphyceae. Rev. Brasil. Biol. 53(3): 477- 488 (in Portuguese, with abstract in English).

Brook, A.J. 1981. The biology of desmids. Blackwell Scientific Publications. Oxford, London. 276 pp.

Coesel, P.F.M. 1996. Biogeography of desmids. Hydrobiologia, 336: 41-53.

De Lamonica- Freire, E. M. 1992. Filamentous Desmids (Zygnemaphyceae, Desmidiales) of Taimã Island Ecological Station, Mato Grosso, Brazil. Acta Limnol. Brasil. Vol: . 4. 315325 (in Portuguese, with abstract in English).

Dias, I. C. A.; Sophia, M. G. 1994. Algae from the State of Goiás and Tocantins, Brazil: Desmidiaceae and Zygnemaceae (Chlorophyta) . Editora da UFG. 54p. (in Portuguese).

Díaz-Castro, J.G.1999. Biomass, biodiversity and abiotic factors drivers of phycoperiphyton bloom in the central channel of the river Jau, at the time of flood, Jau National Park, Central Amazon. PhD Thesis. Instituto Nacional de Pesquisas da Amazônia/Fundação Universidade do Amazonas, Manaus, Amazonas.173pp. (in Portuguese, with abstract in English).

Förster, K. 1969. Amazonian Desmids. 1st part. Area Santarém. Amazoniana, 2: 5-232. (in German with abstract in Portuguese).

Förster, K. 1974. Amazonian Desmids. 2nd part. Area MauésAbacaxis. Amazoniana, 5(2): 135-242. (in German with abstract in Portuguese).

Grönblad, R. 1945. Brazilian algae, specially Desmidiaceae from low region of Amazon river from August Ginzberger samples on year of MCMXXVII. Acta Soc. Sci. Fennicae: série B, 2: 1-42 (in Latin).

Huszar, V.L.M.; Reynolds, C.S. 1997. Phytoplankton periodicity and sequences of dominance in an Amazonian flood-plain lake (Lago Batata, Pará, Brasil): responses to gradual environmental change. Hydrobiologia, 346:169-181.

Junk, W.J.; Bayley, P.B.; Sparks, R.E. 1989. The flood pulse concept in river-flooplain systems. In: D.P. Dodge (ed.). Proceedings of the International Large River Sympisium. Can. Spec. Publ. Fish Aquat. Sci. 106.p. 110-127.

Martins, D.V. 1980. Contribution to phycology in Amazonian, 2: desmid flora of the Cristalino and São Sebastiáo lakes, Amazonas State: filamentous genus. Acta Amazonica 10(4): 725-741 (in Portuguese, with abstract in English).

Melo, S.; Sophia, M. G.; Menezes, M.; Souza, C.A. 2004. Planktonic algae biodiversity from Jaú National Park: Seringalzinho window. In: Borges, S.H.; Iwanaga, S.; Durigan, C.C.; Pinheiro, M.R. (Eds). Windows for biodiversity in Jaú National Park: a strategy for the study of biodiversity in the Amazon. Manaus, Ed. Ipiranga. Brasília. p.83-94. (in Portuguese).
Melo, S.; Rebelo S.R.M.; Souza, K.F.; Soares C.C. Sophia, M.G. 2005. Desmids with planktonic occurrence. In: Santos Silva, E.N.; Aprile, F.M.; Scudeller, V.V.; Melo, S. Biotupé: physical environment, biological and sociocultural diversity of the low Negro river, Central Amazon. Instituto Nacional de Pesquisas da Amazônia, Manaus, Amazonas. p. 99-108. (in Portuguese).

Melo, S.; Souza, K.F. 2009. Annual and interannual fluctuation of desmids species in a black water Amazon floodplain lake (Lago Cutiuaú, Amazonas State, Brazil). Acta Scientiarum-Biological Science. v.31 (3): 235-243 (in Portuguese, with abstract in English).

Mix, M. 1972. The fine structure of Mesotaeniaceae and Gonatozygaceae with a comparison of the wall types of the Conjugatophyceae and over their systematic value. Archivie für Mikrobiologie, 81:197-220 (in German).

Scott, A.M., Grönblad, R.; Croasdale, H.T. 1965. Desmids from the Amazon Basin, Brazil, collected by Dr. H. Sioli. Acta Botanica Fennica 69: 3-93.

Sioli, H. 1950. The water in Amazonia. Forsch. Fortschr., 26: 274280 (in German).

Sophia, M. G.; Silva, L. H. S. 1989. Some considerations about the filamentous Desmids (Zygnemaphyceae) from Northwest Mato Grosso e Southeast Rondônia states, Brazil. Revista Brasileira de Biologia, 49 (4): $493-956$ (in Portuguese, with abstract in English)

Sophia. M. G.; Huszar, V. L. M. 1996. Planktonic desmids of three Amazonian systems (Lake Batata, Lake Mussurá and Trmbetas river), Pará, Brazil. Amazoniana. XIV (1/2): 75-90.

Sophia, M.G. Dias, I.C.A. 1996. Algae from three clear-water environments in the Municipality of Parintins, Amazon State, Brazil: Oedogoniophyceae and Zygnemaphyceae. Hoehnea 23(2): 59-80 (in Portuguese, with abstract in English).

Souza , K. F.;Melo, S.; Almeida, F. F. 2007. Desmids from a floodplainlake of National Park Jaú (Amazonas- Brazil). Rev Brasil. Biociências, 5(2): 24-26 (in Portuguese).

Souza, K. F. 2008. Desmids (Chlorophyta) in a black water floodplain lake from Central Amazonia. Dissertação de Mestrado, Instituto Nacional de Pesquisas da Amazônia/Fundação Universidade do Amazonas, Manaus, Amazonas. 116pp (in Portuguese, with abstract in English).

Thomasson, K. 1955. Studies on South American fresh-water plankton, 3: plankton from Tierra del Fuego and Valdivia. Acta Horti Gotoburgensis 19: 193-225.

Thomasson, K. 1971. Amazonian algae. Mém. Inst. r. Sci. nat. Belg., Sér 10, 86: 1-57.

Uherkovich, G. 1976. Algae from rivers Rio Negro and Rio Tapajós. Amazoniana. 5(4): 465-515 (in German with abstract in Portuguese).

Uherkovich, G.; Schmidt, G.W. 1974. Phytoplankton in the Central Amazonian alluvial lake Lago do Castanho. Amazoniana V (2): 243-283 (in German with abstract in Portuguese).

Recebido em 28/05/2008

Aceito em 17/12/2009 
